\title{
Two new species of Apoloniinae (Acari: Trombiculoidea: Leeuwenhoekiidae) from African small mammals, with a key to the species of the world
}

\author{
Wayne A. Brown
}

College of Tropical Agriculture and Human Resources, University of Hawaii, Honolulu, Hawaii 96822, USA

Key words: Acari, Africa, Apoloniinae, chiggers, Leeuwenhoekiidae, new species, Trombiculoidea

\begin{abstract}
The genus Liuella Wang et Bai, 1992 is transferred from Trombiculidae to Apoloniinae and a new species Liuella monosetosa sp. n. described from specimens taken in Morocco from the hosts Gerbillus sp. and Meriones libycus Lichtenstein. A new species of Straelensia Vercammen-Grandjean et Kolebinova, 1968, Straelensia variocula sp. $\mathrm{n}$. is described from specimens taken in Morocco and Burkina Faso from the hosts Genetta thierryi Matschie, Gerbillus sp., M. libycus and Elephantulus rozeti (Duvernoy). A list of species, hosts and localities and a key to the species of the Apoloniinae of the world are presented.
\end{abstract}

The subfamily Apoloniinae Wharton, 1947 was created to accommodate two monotypic genera containing the species Apolonia tigipioensis Torres et Braga, 1938 and Womersia strandtmanni Wharton, 1947 from South and North America. Since that time, 17 additional species from Africa, Central America, China, Europe, Russia and Pacific Islands have been added. VercammenGrandjean and Kolebinova (1968) revised the Apoloniinae and divided the subfamily into two tribes, the Sauracarellini and the Apoloniini. The species here described fit morphologically into the Apoloniini and bring the totals for that tribe to 14 species in 5 genera and for the Apoloniinae to 19 species in 8 genera. A list of the species, hosts, and localities is provided and the key of Goff (1983) to then known apoloniine taxa, is revised and expanded.

\section{MATERIALS AND METHODS}

Chiggers from small mammal hosts taken from Morocco and Burkina Faso were collected in conjunction with the African Mammal Project (AMP) of the U.S. National Museum / Smithsonian Institution (USNM) in 1968, 1969 and 1970 The program spanned the period of 1961 to 1972 resulting in a massive ectoparasite collection only a small portion of which is reported on here. Captured mammals were killed, identified to genus and when possible to species by mammalogists and support members of the USNM. Pelts were deposited in the USNM and are recorded by accession number. Pelts were examined for ectoparasites and when found were removed and preserved in $70 \%$ ethanol. Slide mounts were later prepared for microscopy using a clearing agent and polyvinyl alcohol mounting media. Examination of chigger specimens collected in those three years of the project has revealed the presence of the three families Trombiculidae, Leeuwenhoekiidae and Walchiidae. This portion of the study is concentrated on the subfamily Apoloniinae of the Leeuwenhoekiidae. The microscopy for this material utilized both phase contrast (Wild Heerbrugo microscope with bright field, dark field and phase contrast lenses, and micrometer and camera attachments) and differential interference microscopy (Olympus BX-51 with Aptronics Macrofield digital camera and Picture Frame software for computer enhancement). Illustrations were made utilizing micrometer measurements and computerized photomicrographs. Accession numbers are not assigned to the chiggers, which are catalogued alphabetically by species, followed by genus, date and collector. The holotypes of the described species have been deposited in the collection of the U.S. National Museum of Natural History / Smithsonian Institution, Washington, D.C., USA. Paratypes are there, as well as in the collection of the Institute of Parasitology, Academy of Sciences of the Czech Republic, České Budějovice (PaÚ) and collection of the author. Terminology and abbreviations follow Goff et al. (1982). All measurements are given in micrometres $(\mu \mathrm{m})$.

\section{RESULTS}

Liuella Wang et Bai, 1992

Type species: Liuella virtus Wang et Bai, 1992

In 1992 Wang and Bai assigned Liuella virtus to Trombiculini of the Trombiculidae comparing it to Miyatrombicula (Miyatrombicula) kochiensis Sasa, Kawashima et Egashira, 1952 and Miyatrombicula (Miyacarus) tokioensis Kumada, 1954 from Japan. Comparison of Ip, scutal and eye placement measurements and morphology and sternal and coxal setation of Liuella virtus with the Miyatrombicula taxa and with members of the Apoloniinae, tribe Apoloniini, indicate that this species more appropriately belongs in the latter grouping and it is here reassigned. The original diagnosis is repeated with emendation to reflect its transfer from Trombiculidae (Trombiculini) to Apoloniinae (Apoloniini) and to encompass measurement differentials required by addition of a new species.

Emended diagnosis. Larvae of Apoloniinae (Apoloniini) B/B/BB-NB/4BS. Galeala nude. Scutum round shield without anterolateral shoulders, with obvious

Address for correspondence: W.A. Brown, College of Tropical Agriculture and Human Resources, University of Hawaii, 2030 Maile Way, Rm. 310, Honolulu, Hawaii 96822, USA. Phone: ++1 808735 4148; Fax: ++1 808956 2428; E-mail: last@lava.net 
papilla-shaped centro-projection on the posterior margin (cordiform scutum). PL far off the scutum (peniscutum). Sensillae filiform, with setules near base and thick branches closely following the setules. Palpal claw 3pronged. Parasubterminala I absent. Mastifemorala, mastitibiala and mastitarsala all absent but with a rather long nude seta on the middle part of basifemur of leg III. Genuala II and III, tibiala III all absent. fCx $=1 / 2 / 1$. $\mathrm{fSt}=2 / 2 / 9-13$. Chelicerae broad at base, strongly recurved, lacking denticles. SIF 4BS-N-3-1000.0000. Ip 414-648.

\section{Liuella monosetosa sp. n.}

Fig. 1

Description. Larvae 4BS-3-N-1000-0000. Idiosoma measuring $150 \times 110$ in unengorged specimen, $310 \times$ 160 in partially engorged holotype. Eyes 2 pairs, on idiosoma, surrounded by epiostracal pleats, anterior 10 in diameter, posterior weak or unapparent. One pair of humeral setae 24-26 long; 96-104 dorsal idiosomal setae 16-23 long, arranged in irregular rows beginning 12-12; 9-13 sternal setae irregularly placed, 10-15 long; 4-8 bilateral intercoxal setae, between coxa II and III, usually 7, arranged 3-4, 10-15 long; 18-20 preanal setae 10-15 long; 30-34 postanal setae 15-17 long. Total idiosomal setae 159-173 in number. Gnathosoma. Palpal setal formula $\mathrm{B} / \mathrm{B} / \mathrm{BBB} / 4 \mathrm{BS}$; palpal claw 3-pronged, 11-12 long; galeala $\mathrm{N}$; cheliceral blade 14-15 long, broad at base, strongly recurved, lacking denticles; gnathobase lightly punctate to squamous at edges, bearing a pair of branched setae. Scutum. Cordiform, smooth or with few puncta, arched crescents above sensillar bases, biconcave anterior margin lacking nasus; posterior margin rounded with acute pointed apex; convex lateral margins; AM base slightly posterior to AL bases; PL setae extrascutal (peniscutum); SB anterior to level of PL bases; $\mathrm{PL}>\mathrm{AL}>\mathrm{AM}$; sensillae flagelliform with basal barbs and branches along entire structure. Scutal standard measurements are tabulated below. Legs. All 7-segmented, terminating in 2 claws with claw-like empodium. Onychotriches absent. Leg measurements are tabulated below. Leg I: coxa with 1 branched seta (1B); trochanter $1 \mathrm{~B}$; basifemur $1 \mathrm{~B}$; telofemur $5 \mathrm{~B}$; genu $4 \mathrm{~B}$, genuala; tibia 8B, 2 tibialae, microtibiala; tarsus 20B, tarsala, microtarsala, subterminala, pretarsala. Leg II: coxa $2 \mathrm{~B}$; trochanter $1 \mathrm{~B}$; basifemur $2 \mathrm{~B}$; telofemur $4 \mathrm{~B}$; genu 3B; tibia 6B, 2 tibialae; tarsus 16B, tarsala. Leg III: coxa 1B; trochanter $1 \mathrm{~B}$; basifemur $2 \mathrm{~B}$; telofemur $3 \mathrm{~B}$; genu $3 \mathrm{~B}$; tibia $6 \mathrm{~B}$; tarsus $13 \mathrm{~B}$.

Standard measurements of type series $(n=9)$ :

\begin{tabular}{l|cccccccc}
\hline & AW & SB & ASB & PSB & SD & AM & AL & PL \\
\hline holotype & 36 & 10 & 12 & 12 & 24 & 7 & 22 & 24 \\
minimum & 31 & 10 & 12 & 12 & 24 & 6 & 15 & 18 \\
maximum & 36 & 13 & 13 & 17 & 30 & 10 & 22 & 25 \\
mean & 34 & 12 & 12 & 14 & 26 & 7 & 19 & 22 \\
\hline
\end{tabular}

\begin{tabular}{cccccccc}
\hline $\mathrm{S}$ & $\mathrm{H}$ & $\mathrm{D}$ & $\mathrm{V}$ & $\mathrm{pa}$ & $\mathrm{pm}$ & $\mathrm{pp}$ & $\mathrm{Ip}$ \\
\hline 18 & 26 & 18 & 11 & 149 & 124 & 144 & 412 \\
13 & 24 & 16 & 10 & 147 & 122 & 136 & 405 \\
18 & 26 & 23 & 15 & 157 & 126 & 150 & 423 \\
15 & 20 & 20 & 12 & 154 & 124 & 143 & 414 \\
\hline
\end{tabular}

H o s t s : Gerbillus sp., Meriones libycus Lichtenstein.

$\mathrm{T}$ y p e d a t a : Type series from Morocco. Holotype from Gerbillus sp., USNM 473444, Tarfaya, Tuisgui Remz, $28^{\circ} 28^{\prime}$ N, $09^{\circ} 12^{\prime}$ W, 15 XII 1969, Col. L. W. Robbins. Site habitat was a small valley wash with gravel, soil and some vegetation, surrounded by small rocky hills. Eight paratypes, number from each host in parentheses. Gerbillus sp., USNM 472247 (1), Agadir, Aouinet Torkoz, 28² 'N, $09^{\circ} 52^{\prime} \mathrm{W}, 07$ XI 1969, Col. H. W. Setzer. Site habitat was a small palm grove near rocky hillside. USNM 471732 (2), Ouarzazate, Foum Zguid, $30^{\circ} 04^{\prime} \mathrm{N}, 06^{\circ} 53^{\prime} \mathrm{W}, 07$ III 1970, Col. M. G. Hearst. Site habitat was at oasis palm grove edge with acacias and pebble desert. USNM 471736 (1), Ouarzazate, Foum Zguid, 3004'N, 06 $53^{\circ}$ 'W, 07 III 1970, Col. M. G. Hearst. Site habitat as above. USNM 471780 (1), Ouarzazate, Foum Zguid, $30^{\circ} 04^{\prime} \mathrm{N}, 06^{\circ} 53^{\prime} \mathrm{W}, 07$ III 1970, Col. M. G. Hearst. Site habitat as above. Meriones libycus, USNM 473913 (3), Ouarzazate, Tazenakht $30^{\circ} 04^{\prime} \mathrm{N}, 07^{\circ} 17^{\prime} \mathrm{W}$. Site habitat consisted of fairly thick vegetation of thorn bushes and small woody shrubs in hills above a riverbed. The holotype and six paratypes (M-1 to $\mathrm{M}-7)$ are deposited in USNM, one paratype (M-8) is in the collection of the author and one paratype (M-9) is in PaÚ (coll. no.: PaÚ 2005).

E t y m o 1 o g y : The new species name "monosetosa" reflects the presence of a single anterior medial seta characteristic of this genus.

Differential diagnosis. Liuella monosetosa can be separated from the type species $L$. virtus by the smaller size and standard data measurements Ip $=648$, AW $=$ $45, \mathrm{SD}=51$ in that species as opposed to Ip $=414, \mathrm{AW}$ $=34, \mathrm{SD}=26$ in L. monosetosa. It further differs from L. virtus by the less pilose total idiosomal setae, circa 253 in that species while $L$. monosetosa displays circa 170.

\section{Straelensia Vercammen-Grandjean et Kolebinova, 1968}

Type species: Straelensia europaea Vercammen-Grandjean et Kolebinova, 1968

The original diagnosis, based on one specimen, was emended by Wen et al. (1996) in their paper which encompassed properties of new members assigned to Straelensia. This new species fits within the parameters of that re-diagnosis.

\section{Straelensia variocula $\mathrm{sp} . \mathrm{n}$.}

Fig. 2

Description. Larvae 4BS-3-N-1000-0000. Idiosoma measuring $500 \times 270$ in partially engorged specimen. Eyes 2 pairs, on idiosoma surrounded by epiostracal pleats, anterior 10 in diameter, posterior weak, often absent or unapparent. One pair of humeral setae 34-38 

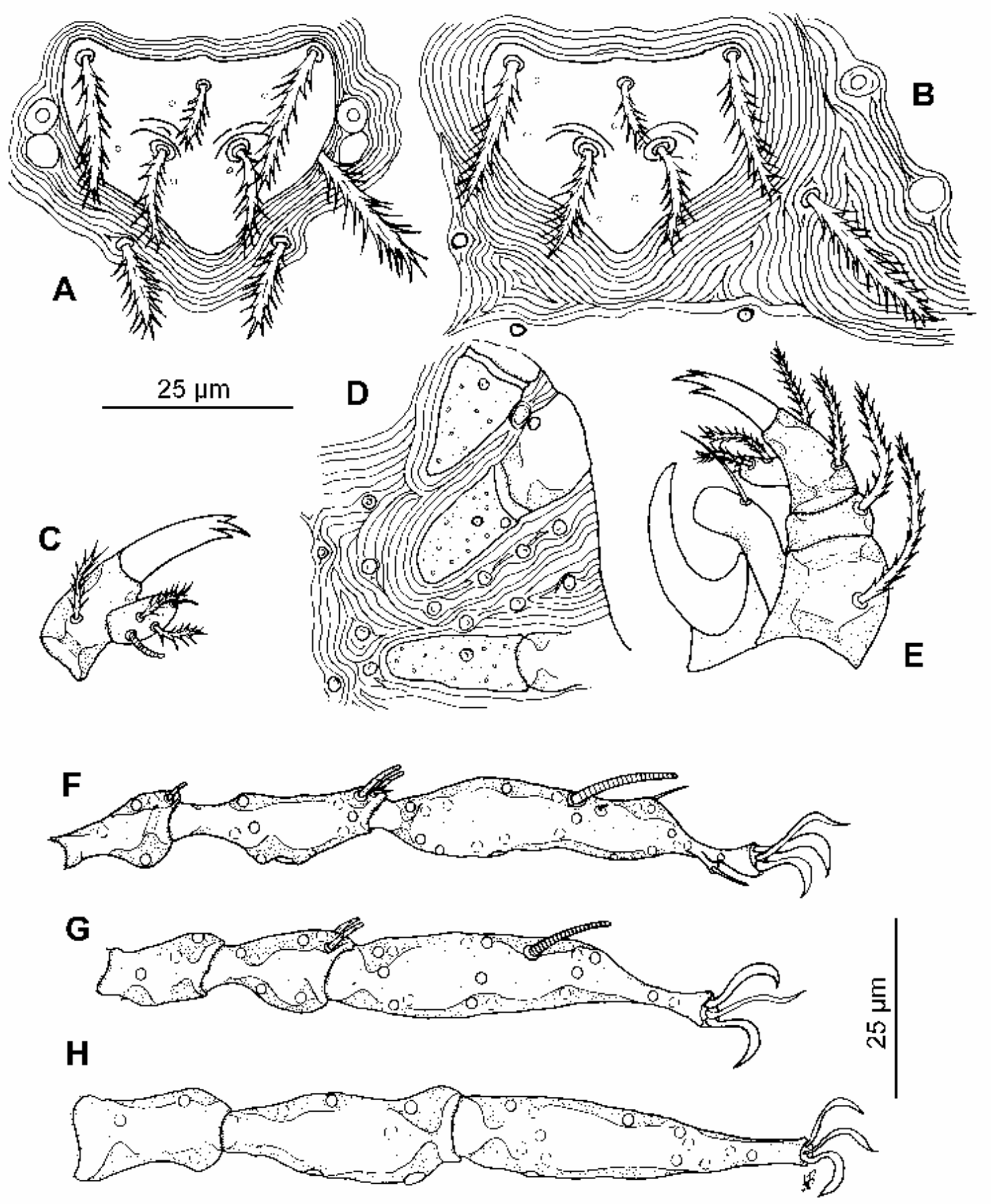

Fig. 1. Liuella monosetosa sp. n. A - Scutal area of unengorged specimen with placement of eyes, extrascutal PLs and 2 dorsal setae. B - Scutal area of partially engorged specimen, as above. C - Ventral aspect of palpal tarsus. D - Sternal area with placement of coxal setae, and sternal and intercoxal setae, both of which are variable in placement and number. $\mathbf{E}-$ Dorsal aspect of gnathosoma. F - Leg I distal three segments showing specialised setae and bases of branched setae. $\mathbf{G}-$ Leg II, as above. $\mathbf{H}-$ Leg III, as above.

long; 96-110 dorsal idiosomal setae 20-28 long, arranged in irregular rows beginning $12-12 ; 7-13$ sternal setae irregularly placed, 16-20 long; 5-9 bilateral intercoxal setae between coxa II and III, usually placed 4-3, 16-20 long; 46-50 preanal setae 16-20 long; 46-50 postanal setae 16-20 long; total idiosomal setae 202234 in number. Gnathosoma. Palpal setal formula B/B/BBB/4BS; palpal claw 3-pronged, 16-20 long; galeala N; cheliceral blade 15-16 long, broad at base, strongly recurved without denticles; gnathobase lightly 
punctate to squamous at edges, bearing a pair of branched setae. Scutum. Cordiform, smooth or with few puncta, arched crescents above sensillar bases, with biconcave anterior margin and anteriomedial nasus $3 \times$ 10 long; posterior margin rounded with acute pointed apex; convex lateral margins; AM base slightly posterior to $\mathrm{AL}$ bases; PL setae extrascutal; $\mathrm{SB}$ anterior to level of PL bases; PL>AL >AM; sensillae flagelliform with basal barbs and branches throughout structure. Scutal measurements are tabulated below. Legs. All 7segmented, terminating in 2 claws with claw-like empodium. Onychotriches absent. Leg measurements are tabulated below. Leg I: coxa with 1 branched seta (1B); trochanter 1B; basifemur 1B; telofemur $5 \mathrm{~B}$; genu $4 \mathrm{~B}$, genuala; tibia 8B, 2 tibialae, microtibiala; tarsus 20B, tarsala, microtarsala, pretarsala. Leg II: coxa $2 \mathrm{~B}$; trochanter 1B; basifemur 1B; telofemur 5B; genu 3B; tibia 5B, 2 tibialae; tarsus 16B, tarsala. Leg III: coxa 1B; trochanter $1 \mathrm{~B}$; basifemur $1 \mathrm{~B}$; telofemur $4 \mathrm{~B}$; genu $3 \mathrm{~B}$; tibia 6B; tarsus $13 \mathrm{~B}$.

Standard measurements of type series $(n=15)$ :

\begin{tabular}{l|cccccccc}
\hline & AW & SB & ASB & PSB & SD & AM & AL & PL \\
\hline holotype & 32 & 15 & 18 & 19 & 37 & 9 & 24 & 29 \\
minimum & 32 & 12 & 15 & 15 & 30 & 6 & 18 & 27 \\
maximum & 44 & 17 & 20 & 22 & 42 & 10 & 24 & 36 \\
mean & 36 & 15 & 17 & 19 & 36 & 7 & 21 & 30 \\
\hline
\end{tabular}

\begin{tabular}{cccccccc}
\hline S & H & D & V & pa & pm & pp & Ip \\
\hline 20 & 36 & 22 & 18 & 168 & 150 & 162 & 492 \\
18 & 34 & 20 & 16 & 165 & 146 & 152 & 480 \\
27 & 38 & 28 & 20 & 186 & 165 & 184 & 530 \\
22 & 36 & 24 & 18 & 174 & 174 & 168 & 505
\end{tabular}

H o s t s : Elephantulus rozeti (Duvernoy), Genetta thierryi Matschie, Gerbillus sp., Meriones libycus Lichtenstein.

T y p e d a t a : Type series from Morocco and Burkina Faso. Morocco: Holotype from Elephantulus rozeti, USNM 476133, Fes Missour, 330'N, 0405'W, 28 VI 1970, Col. L. W. Robbins. Site habitat was a bushy wash area. Fourteen paratypes, number from each host in parentheses. Elephantulus rozeti, USNM 476133 (2), Fes Missour, $33^{\circ} 08^{\prime} \mathrm{N}, 04^{\circ} 05^{\prime} \mathrm{W}, 28$ VI 1970, Col. L. W. Robbins. Site as above. Gerbillus sp., USNM 472768 (2), Ouarzazate, Ouarzazate, $30^{\circ} 52^{\prime} \mathrm{N}, 06^{\circ} 52^{\prime} \mathrm{W}, 23$ II 1970 , Col. L. W. Robbins. Site habitat was sandy wash with rocky hillsides with little vegetation. USNM 472769 (1), Ouarzazate, Ouarzazate, $30^{\circ} 52^{\prime} \mathrm{N}, 06^{\circ} 52^{\prime} \mathrm{W}, 23$ II 1970 , Col. L. W. Robbins. Site as above. USNM 472774 (1), Ouarzazate, Ouarzazate, $30^{\circ} 52^{\prime} \mathrm{N}, 06^{\circ} 52^{\prime} \mathrm{W}, 24$ II 1970 , Col. L. W. Robbins. Site as above. USNM 472772-3 (1), Ouarzazate, Ouarzazate, $30^{\circ} 52^{\prime} \mathrm{N}, 06^{\circ} 52^{\prime} \mathrm{W}, 23$ II 1970 , Col. L. W. Robbins. Site as above. USNM 472778 (1), Ouarzazate, Ouarzazate, $30^{\circ} 52^{\prime} \mathrm{N}, 06^{\circ} 52^{\prime} \mathrm{W}, 23$ II 1970 , Col. L. W. Robbins. Site as above. USNM 471968 (1) Oujda, Figuig, $32^{\circ} 09^{\prime} \mathrm{N}, 01^{\circ} 15^{\prime} \mathrm{W}, 09$ II 1970 , Col. M. G. Hearst. Site habitat was rocky, sand dunes with vegetation tufts. USNM

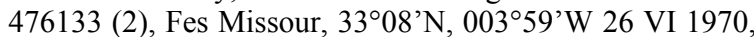
Col. L. W. Robbins. Site habitat rocky plain. Meriones

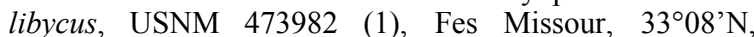
$003^{\circ} 59^{\prime}$ W, 26 VI 1970, Col. L. W. Robbins. Site as above.
Burkina Faso: Genetta thierryi, USNM 450962 (2). Natiaboni, $11^{\circ} 42^{\prime} \mathrm{N}, 00^{\circ} 30^{\prime} \mathrm{E}, 11$ XII 1968, Col. R. E. Vaden. Site habitat riverbed, no running water but with some pools and areas of high grass. The holotype and twelve paratypes (M1 to $\mathrm{M}-11$ and $\mathrm{B}-1$ to $\mathrm{B}-2)$ are deposited in USNM, one paratype (M-12) is in the collection of the author and one paratype (M-13) is in PaÚ (coll. no.: PaÚ 2006).

E t y m o lo g y: The species name reflects the variability of observing the posterior eyes of this taxon.

Differential diagnosis. Straelensia variocula can be separated from $S$. europaea, by the smaller size and standard data measurements Ip $=676, \mathrm{SD}=48$ in that species as opposed to Ip $=505, \mathrm{SD}=36$ in $S$. variocula and the differing galeala seta, branched in $S$. europaea but nude in $S$. variocula. It differs from $S$. africana by the more pilose total idiosomal setae, circa 130 in that species while $S$. variocula displays circa 218 and in the differing alignment of coxalaea II, parallel in S. africana and perpendicular in the new species.

\section{Key to the species of the Apoloniinae of the world}

1 Sensillae expanded ............................... 2

- Sensillae slender. 4

2 Coxa II bisetose; palpal tarsus 6BS; 3 galealae I ... Afracarella africana

- Coxa II unisetose; palpal tarsus 7BS; 2 galealae I .................................................... 3

3 Tarsala I $<$ Tarsala II; AM $<$ PL Sauracarella whartoni

- Tarsala I > Tarsala II; AM >PL

Sauracarella montana

4 Nasus present ..................................... 5

- Nasus absent ..................................... 16

5 Single AM seta .................................. 6

- Paired AM setae ............................... 11

6 Palpal tarsus formula 5B or greater .............. 7

- Palpal tarsus formula 4BS or less .................. 10

7 Palpal tarsus formula 5B; large nasus with AM seta on nasus ................................. 8

- Palpal tarsus formula 5BS or greater; thin nasus with AM seta on scutum ........................ 15

8 Tarsala II bluntly tapering ........................ ......................... Womersia strandtmanni

- Tarsala II with bulbapex. ......................... 9

9 Mastitarsala III absent; eyes on ocular plate......... Womersia irani

- Mastitarsala III present; eyes on idiosoma .......... Womersia midwayensis

10 Palpal tarsus 3BNS; palpal tibia 2B and with a subterminal 3-pronged dorsal curved spine. Straelensia cynotis

- Palpal tarsus 4BS; palpal tibia 3B ................ 11

11 Galeala B ....................................... 12

- Galeala N ...................................... 14 

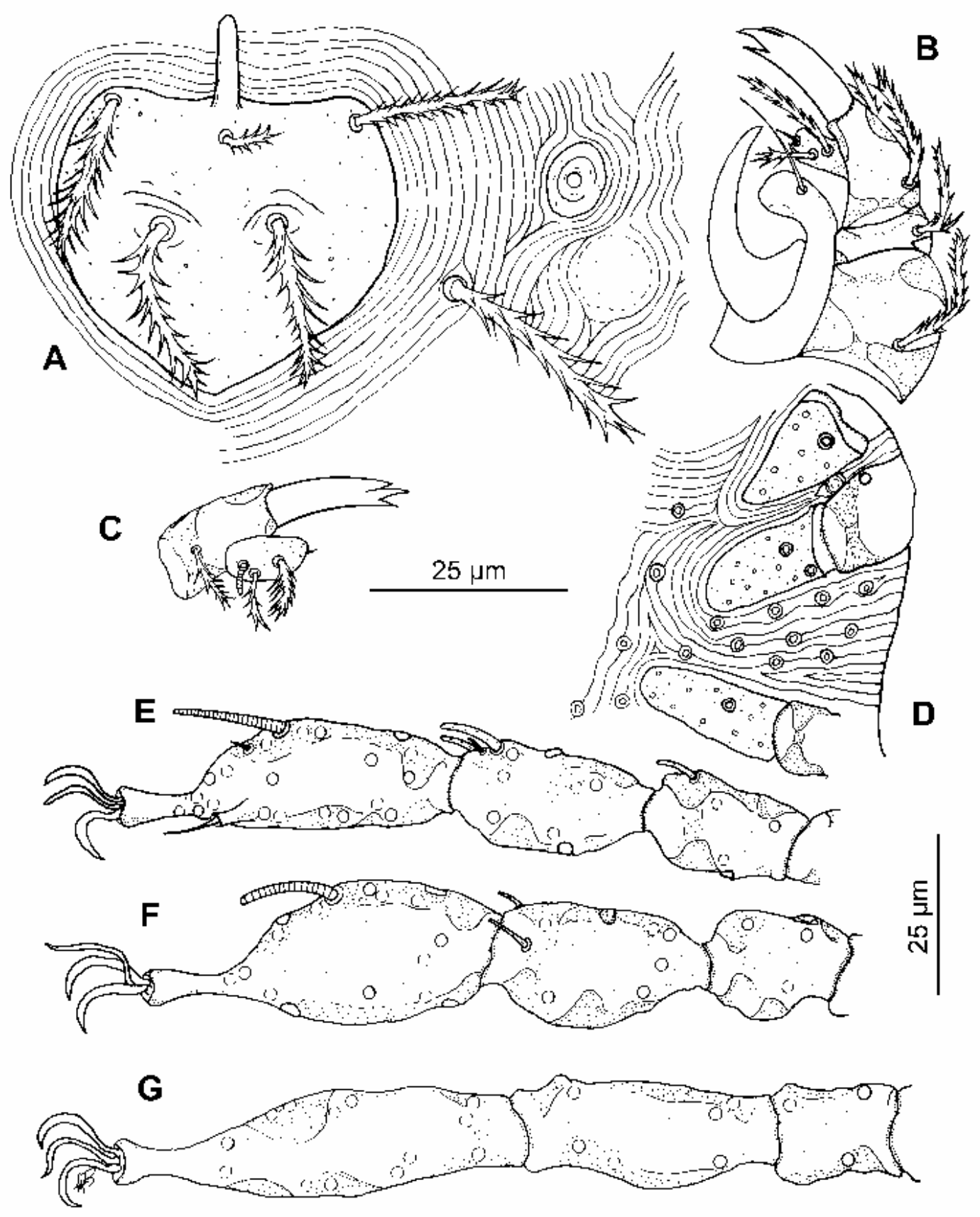

Fig. 2. Straelensia variocula sp. n. A - Scutal area with placement of eyes and extrascutal PLs. B - Dorsal aspect of gnathosoma. C - Ventral aspect of palpal tarsus. D - Sternal area with placement of coxal setae, and sternal and intercoxal setae, both of which are variable in placement and number. E - Leg I distal three segments showing specialised setae and bases of branched setae. F - Leg II, as above. G - Leg III, as above.

12 Index pedibus greater than 600

- Index pedibus less than 580

.. Straelensia taurica

13 Coxalaea III centrally located; NDV $\approx 208$ Straelensia europaea

- Coxalaea III excentrally located, anteriorly; NDV $\approx 239$ Straelensia tiani
14 Coxalaea II parallel to edge of segment; NDV $\approx$ 130 .......................... Straelensia africana

- Coxalaea II perpendicular to edge of segment; $\mathrm{NDV} \approx 218$............. Straelensia variocula sp.n.

15 Palpal tarsus formula 5BS; subterminala absent ... Afropolonia tgifi

- Palpal tarsus formula 7BS; subterminala present ... Apolonia tigipioensis 
16 Single AM seta; coxal setae II bisetose

- Paired AM setae; coxal setae II unisetose or bisetose

17 Index pedibus $\approx 648 ;$ NDV $\approx 253$ Liuella virtus

- Index pedibus $\approx 414 ;$ NDV $\approx 170$ Liuella monosetosa sp.n.

18 Coxa II bisetose; tibiala III absent; eyes absent .... Vargatula hispida

- Coxa II unisetose; tibiala III present; eyes present 19
19 Pretarsala II present; palpal claw 4-pronged ........ Vargatula somaliensis

- Pretarsala II absent; palpal claw 3-pronged Vargatula pacifica

Acknowledgements. Thanks are extended to two anonymous reviewers for critically reading and providing useful comments on the manuscript. Studies were conducted at the College of Tropical Agriculture and Human Resources, University of Hawaii, Honolulu, Hawaii.

Table 1. Species of the Apoloniinae of the world.

\begin{tabular}{|c|c|c|}
\hline Chigger species & Host species & Localities \\
\hline Afracarella africana (Lawrence, 1949) & Tetradactylus seps seps & South Africa \\
\hline Sauracarella whartoni Lawrence, 1949 & Pseudocordylus subviridis & South Africa \\
\hline Sauracarella montana Lawrence, 1949 & Tropidosaura cottrelli & South Africa \\
\hline Womersia strandtmanni Wharton, 1947 & Pelecanus occidentalis & North America \\
\hline $\begin{array}{l}\text { Womersia irani Vercammen-Grandjean, } \\
\text { Rhode et Mesgali, } 1970\end{array}$ & Neophron percnopterus & Iran \\
\hline Womersia midwayensis Goff, Sivert et Sileo, 1989 & Diomedea immutabilis & Midway Island \\
\hline $\begin{array}{l}\text { Straelensia europaea Vercammen-Grandjean } \\
\text { et Kolebinova, } 1968\end{array}$ & Canis lupus & Bulgaria \\
\hline Straelensia africana Vercammen-Grandjean, 1971 & Herpestes sanguineus & South Africa \\
\hline Straelensia taurica Hushcha, 1975 & Lepus europaeus & Crimea, Ukraine \\
\hline Straelensia tiani Wen, Tian, Guan et Wang, 1996 & Lepus capensis & Shanxi, China \\
\hline Straelensia cynotis Fain et Le Net, 2000 & Canis sp., dogs & France \\
\hline Straelensia variocula sp. $\mathrm{n}$. & $\begin{array}{l}\text { Elephantulus rozeti, Genetta thierryi, } \\
\text { Gerbillus sp., Meriones libycus }\end{array}$ & $\begin{array}{l}\text { Morocco and Burkina } \\
\text { Faso, Africa }\end{array}$ \\
\hline Afropolonia tgifi Goff, 1983 & Aethomys namaquensis & South Africa \\
\hline Apolonia tigipioensis Torres et Braga, 1938 & Gallus gallus domesticus, man & South America \\
\hline Vargatula hispida Brennan et Yunker, 1966 & Dasypus novemcinctus & Panama, C. America \\
\hline Vargatula pacifica Goff et Loomis, 1982 & Sus scrofa & N. Solomon Islands \\
\hline Vargatula somaliensis Goff, 1983 & Heterocephalus glaber & Somalia, Africa \\
\hline Liuella virtus Wang et Bai, 1992 & Citellus dauricus alashanicus & Ningxia, China \\
\hline Liuella monosetosa sp. n. & Gerbillus sp., Meriones libycus & Morocco, Africa \\
\hline
\end{tabular}

\section{REFERENCES}

GOFF M.L. 1983: A new genus and species of Apoloniinae (Acari: Trombiculidae) from South Africa with a key to the species in the subfamily. Bull. Inst. R. Sci. Nat. Belg. 55(9): 1-9.

GOFF M.L., LOOMIS R.B., WELBOURN W.C., WRENN W.J. 1982: A glossary of chigger terminology ( Acari: Trombiculidae). J. Med. Entomol. 19: 221-238.

VERCAMMEN-GRANDJEAN P.H. 1971: Straelensia africana (Acarina, Prostigmata), a new representative of the Apoloniinae subfamily. Z. Angew. Zool. 35: 99-103.

VERCAMMEN-GRANDJEAN P.H., KOLEBINOVA M. 1968: Revision of the subfamily Apoloniinae Wharton,
1947 (Leeuwenhoekiidae: Acarina). Acarologia 19: 250258.

WANG D.-Q., BAI X.-L. 1992: A new genus of chigger from Ningxia, China (Acari: Trombiculidae). Acta Entomol. Sin. 35: 247-249.

WEN T.-H., TIAN Q.-U., GUAN Y., WANG W.-L. 1996: First record of Apoloniinae in China - Straelensia tiani sp. n. - with a revised diagnosis of the genus Straelensia (Acariformes: Leeuwenhoekiidae). Acarologia 37: 211215.

Accepted 13 March 2006 